\title{
Uso de parámetros sedimentológicos en el reconocimiento de ambientes hidrodinámicos en los canales y fiordos adyacentes a «Campos de Hielo Sur»
}

\author{
Felipe Ríos R. ${ }^{1}$ y Marco Cisternas V. ${ }^{2}$ \\ ${ }^{1}$ Departamento de Oceanografía, Universidad de Concepción, Casilla 150-C, Concepción, Chile. \\ E-mail: frios@udec.cl \\ ${ }^{2}$ Centro EULA-Chile, Universidad de Concepción, Casilla 156-C, Concepción, Chile. \\ E-mail: mcisterv@udec.cl
}

\begin{abstract}
RESUMEN. Durante el crucero de investigación científica marina a los fiordos de Campos de Hielo Sur, abordo del buque oceanográfico AGOR "Vidal Gormaz", se obtienen muestras sedimentológicas con un Box-corer en 17 estaciones. Se estudian los fondos blandos de los fiordos y canales adyacentes al sistema glacial. Se realiza una caracterización sedimentológica, reconociendo los principales factores reguladores del proceso sedimentario a través de parámetros texturales y granulométricos. Predominan las texturas limosas, con distribuciones granulométricas moderadamente seleccionadas, asimetrías negativas y leptocúrticas. Sobre la base de la distribución espacial de los parámetros sedimentológicos, el sistema de fiordos y canales se divide en dos zonas de energía: (a) zona de baja energía en las cabeceras protegidas; y (b) zona de mayor energía en los canales expuestos. La materia orgánica total y coloración de los sedimentos indican que el área está sujeta a una renovación periódica de sus aguas y que los materiales orgánicos provienen del ambiente marino. Se reconoce que el canal Messier, paso del Indio, bocas de los senos Penguin y Europa son una unidad sedimentaria, probablemente controlada por las corrientes de mareas.
\end{abstract}

Palabras claves: Chile austral, Campos de Hielo Sur, fiordos, sedimentología, parámetros granulométricos, hidrodinamismo.

\section{Use of grain size parameters in the recognition of hydrodynamic environments in the fjord inlets adjacent to «Campos de Hielo Sur»}

\begin{abstract}
Seventeen soft bottom sediment samples taken from the fjord inlets adjacent to the glacial system of «Campos de Hielo Sur», with a box-corer, from the research vessel AGOR "Vidal Gormaz", are analysed. A sedimentological characterization was conducted, resulting in the recognition of the main factors controlling the sedimentary process through the use of textural and grain-size parameters. It was found that most of the area is covered by silty sediments. They are moderately sorted, negatively skewed and leptokurtic. The spatial distribution of these parameters point out to the existence of two sedimentary environments with different levels of energy (sheltered heads and exposed inlets). The total organic matter and color of the sediments show that the area is subjected to periodic renewals of the bottom waters and that the organic matter is been supplied from the marine environment. Canal Messier, paso del Indio, the mouth of the Penguin and Europe bays are recognized as being part of one sedimentary unit, probably regulated by the action of tidal currents.
\end{abstract}

Key words: Southern Chile, Campos de Hielo Sur, fjords, sedimentology, grain size parameters, hydrodynamism.

\section{INTRODUCCION}

Durante los últimos años, el sistema glaciar denominado "Campos de Hielo Sur" ha sido el centro de la atención pública. Esta situación ha desencadenado un creciente interés por parte de la comunidad científica chilena, por estudiar y comprender el funcionamiento de este sistema natural ubicado en la región austral del país.

Campos de Hielo Sur es el mayor sistema glacial de zonas templadas del hemisferio sur, con una extensión de $350 \mathrm{~km}$ de largo por $60 \mathrm{~km}$ de ancho. De acuerdo a imágenes satelitales, cuenta con una superficie de aproximadamente $13.000 \mathrm{~km}^{2}$ (CONA, 1996). Los Campos de Hielo Sur se conectan con el océano Pacífico a través de una compleja red de canales y fiordos que presentan rasgos peculiares, respecto al resto de la costa chilena, caracterizada principalmente por una morfología rectilínea, producto de la colisión de placas.

Los fiordos y canales son entradas de la costa excavadas por la acción del hielo. Generalmente, su cabecera está en contacto directo con el glaciar y su boca está abierta al intercambio, directo o indi- 
recto, con el mar. Transversalmente, su profundo valle tiene forma de «U», terminando longitudinalmente con un umbral submarino formado por las morrenas terminales. Por sus características morfológicas, los fiordos presentan una circulación típicamente estuarina. Sin embargo, la profundidad del umbral puede afectar el intercambio de agua con el océano, modificando su circulación, restringiendo la entrada de agua salina profunda y provocando el estancamiento de ésta (Pritchard, 1976).

Los sistemas de fiordos representan uno de los más importantes rasgos geomorfológicos costeros en las altas latitudes, pues debido a sus características circulatorias y morfológicas, controlan en gran medida el flujo de sedimentos terrígenos hacia el mar. La mayor parte del material producido por acción glacial, es retenido por estos ambientes semicerrados a través de la sedimentación. Por lo tanto, los fondos blandos de los fiordos son ambientes influidos por una compleja combinación de fuentes sedimentarias, caudales fluviales controlados por los deshielos, mareas, corrientes de marea y oleaje.

El sistema de fiordos y canales de Campos de Hielo Sur ha sido estudiado desde principios de los años setenta (Pickard, 1971), sin embargo la mayoría de las investigaciones se han orientado principalmente al estudio de las características oceanográficas de sus aguas. De este modo, no se han realizado hasta la fecha caracterizaciones sedimentológicas de gran escala espacial en los fondos blandos de este sistema glaciomarino.

De acuerdo con lo anterior, el objetivo de la presente investigación es realizar una caracterización sedimentológica en los fiordos y canales de los Campos de Hielo Sur, reconociendo los principales factores reguladores de la distribución sedimentaria a través de los parámetros texturales y granulométricos.

\section{MATERIALES Y METODOS}

\section{Area de estudio}

La región de los fiordos y canales adyacentes a Campos de Hielo Sur (Fig. 1), se extiende aproximadamente desde los $48^{\circ} \mathrm{S}$ (al sur del golfo de Penas) hasta los $52^{\circ} \mathrm{S}$ (al norte del estrecho de Magallanes). $\mathrm{El}$ área es resultado del intenso trabajo de los hielos andinos que han seccionado al relieve por medio de numerosos fiordos. Simultáneamente, la región ha sido sometida a una tectónica de hundimiento a es-

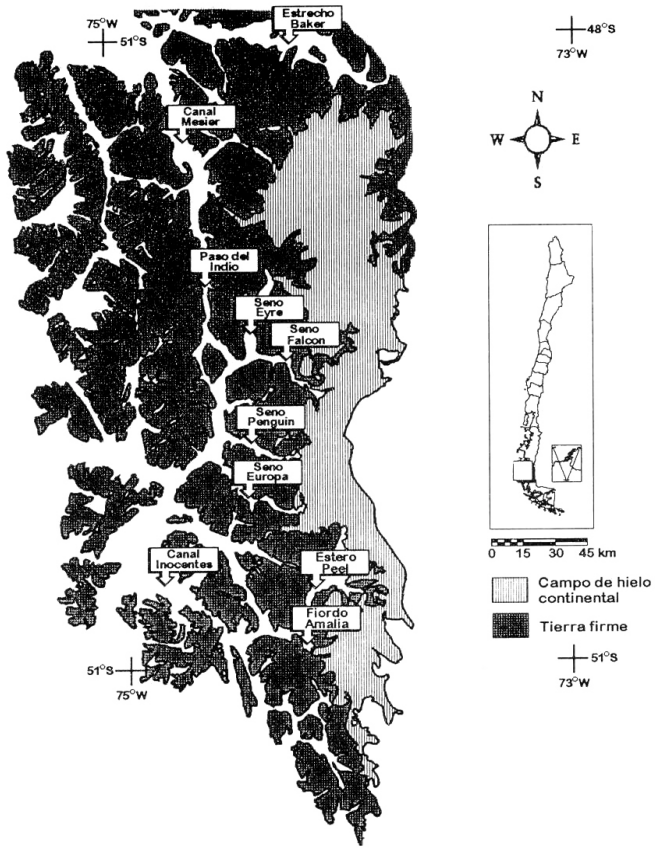

Figura 1. Esquema del sistema de fiordos y canales adyacentes a "Campos de Hielo Sur".

Figure 1. Sketch map of the fjord inlet system adjacent to "Campos de Hielo Sur".

cala geológica, permitiendo el ingreso del mar por los valles occidentales inferiores (Börgel, 1983). Esta transgresión marina generó variadas formas litorales (golfos, canales, senos, estuarios y fiordos) que separan islas y archipiélagos (Börgel, 1983). Longitudinalmente, esta costa se puede separar en dos zonas: hacia el oriente, las geoformas están representadas por las cabeceras protegidas de los valles inundados con directa influencia glaciar, y hacia el occidente, los canales y fiordos son más abiertos y expuestos al accionar marino.

El clima del sector está regulado por la presencia del frente polar y los vientos predominantes del oeste (con velocidades máximas $>100 \mathrm{~km} / \mathrm{h}$ ). El área registra una baja insolación con temperaturas inferiores a $10^{\circ} \mathrm{C}$. El promedio anual de la precipi- 
tación $(3000 \mathrm{~mm})$ está determinado por el relieve y la influencia marina. Estas condiciones generan un clima templado frío lluvioso sin estación seca (González et al., 1987).

La región está influenciada superficialmente por los flujos de aguas antárticas, con temperaturas que oscilan entre $-1,8^{\circ} \mathrm{C}$ y $-1,0^{\circ} \mathrm{C}$. La salinidad fluctúa entre 34 y 35 psu, alcanzando en la estación estival, debido al derretimiento de la capa de hielo y a la escorrentía resultante, valores inferiores a 33 psu (González et al., 1987). El clima de olas se caracteriza por oleaje de gran altura, corta longitud de onda $\mathrm{y}$ alta pendiente, controlado por los variables y fuertes vientos del frente polar. El rango de la marea de sicigias es de aproximadamente $2 \mathrm{~m}$ (SHOA, 1996).

\section{Obtención y análisis de las muestras}

Entre agosto y septiembre de 1995, abordo del buque AGOR "Vidal Gormaz" de la Armada de Chile, se efectuaron 17 estaciones de muestreo en la parte central de los canales y fiordos adyacentes a Campos de Hielo Sur, obteniéndose 17 muestras sedimentológicas (Fig. 2; Tabla 1). La ubicación de cada estación se logró mediante un navegador satelital (GPS). La extracción de las muestras se efectuó con un Box-corer modelo Reineck con una superficie de dragado de $400 \mathrm{~cm}^{2}$, muestreándose los primeros $10 \mathrm{~cm}$ de sedimento.

De cada muestra se obtuvieron dos submuestras representativas. La primera se utilizó para calcular

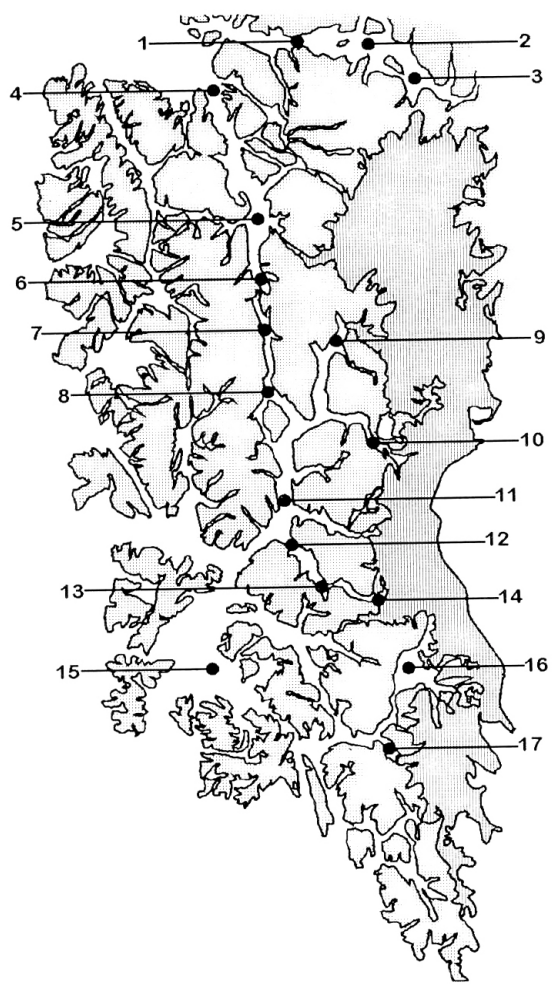

Figura 2. Ubicación de las estaciones de muestreo. Figure 2. Location of sampling stations.

Tabla 1. Ubicación geográfica y profundidad de las estaciones de muestreo.

Table 1. Location and depth of sampling stations.

\begin{tabular}{|ccccc|}
\hline Estación & Lugar geográfico & Latitud $(\mathbf{S})$ & Longitud $(\mathbf{W})$ & Profundidad (m) \\
\hline 1 & Estrecho Baker & $47^{\circ} 58,6^{\prime}$ & $74^{\circ} 14,7^{\prime}$ & 734 \\
2 & Estrecho Baker & $47^{\circ} 59,5^{\prime}$ & $73^{\circ} 47,0^{\prime}$ & 1066 \\
3 & Estrecho Baker & $48^{\circ} 00,7^{\prime}$ & $73^{\circ} 37,3^{\prime}$ & 382 \\
4 & Canal Messier & $48^{\circ} 07,2^{\prime}$ & $74^{\circ} 36,8^{\prime}$ & 684 \\
5 & Canal Messier & $48^{\circ} 42,1^{\prime}$ & $74^{\circ} 24,8^{\prime}$ & 348 \\
6 & Canal Messier & $48^{\circ} 54,4^{\prime}$ & $74^{\circ} 23,9^{\prime}$ & 367 \\
7 & Paso del Indio & $49^{\circ} 02,5^{\prime}$ & $74^{\circ} 26,0^{\prime}$ & 236 \\
8 & Paso del Indio & $49^{\circ} 22,4^{\prime}$ & $74^{\circ} 25,4^{\prime}$ & 283 \\
9 & Seno Eyre & $49^{\circ} 20,2^{\prime}$ & $74^{\circ} 04,4^{\prime}$ & 363 \\
10 & Seno Falcon & $49^{\circ} 38,7^{\prime}$ & $73^{\circ} 49,6^{\prime}$ & 487 \\
11 & Seno Penguin & $49^{\circ} 53,7^{\prime}$ & $74^{\circ} 19,8^{\prime}$ & 453 \\
12 & Seno Europa & $50^{\circ} 07,3^{\prime}$ & $74^{\circ} 13,8^{\prime}$ & 167 \\
13 & Seno Europa & $50^{\circ} 12,7^{\prime}$ & $74^{\circ} 04,4^{\prime}$ & 186 \\
14 & Seno Europa & $50^{\circ} 14,4^{\prime}$ & $74^{\circ} 01,6^{\prime}$ & 45 \\
15 & Canal Inocentes & $50^{\circ} 34,1^{\prime}$ & $74^{\circ} 45,5^{\prime}$ & 204 \\
16 & Estero Peel & $50^{\circ} 31,3^{\prime}$ & $73^{\circ} 44,9^{\prime}$ & 150 \\
17 & Fiordo Amalia & $50^{\circ} 52,1^{\prime}$ & $73^{\circ} 51,2^{\prime}$ & \\
\hline
\end{tabular}


el contenido de materia orgánica total (MOT), secándola a $60^{\circ} \mathrm{C}$ durante $72 \mathrm{hr}$ y luego incinerándola a $550^{\circ} \mathrm{C}$ por $4 \mathrm{hr}$. Los porcentajes fueron calculados por diferencia de peso una vez finalizado el procedimiento (Mills, 1978). Mientras que la segunda se utilizó para analizar la granulometría, se dispersó disuelta en electrolito, mediante un tratamiento de ultrasonido por un período de 5 minutos y posteriormente, se obtuvo la distribución granulométrica empleando un Analizador Electrónico de Micropartículas Elzone 282PC.

El diámetro medio y los parámetros de dispersión (desviación estándar, asimetría y curtosis) se calcularon por el método de momentos (SewardThompson y Hails, 1973). Los datos se representaron en la escala logarítmica phi ( $f$ ) para realizar la descripción del sedimento mediante numeración entera:

$$
f=-\log _{2} D
$$

donde: $\mathrm{D}=$ diámetro de la partícula en $\mathrm{mm}$

Utilizando las frecuencias porcentuales de cada intervalo de clase, se construyeron histogramas de frecuencia. De acuerdo al tamaño medio de la distribución, las muestras se clasificaron texturalmente (Wentworth, 1922). Finalmente, mediante una Carta de colores (Goddard, 1975), se clasificaron los sedimentos según su coloración.

\section{RESULTADOS}

El área de estudio se caracterizó por la presencia mayoritaria de fracciones finas en el sedimento, específicamente limo muy fino y arcilla. De las dos clases texturales reconocidas, el limo muy fino fue el componente predominante en las facies texturales, es decir, presentó la mayor distribución superficial en los fiordos y canales estudiados. Por otra parte, no se observó la presencia de fracciones más gruesas (e.g. arena y grava).

La Tabla 2 muestra los porcentajes de los componentes texturales en los sedimentos de las 17 estaciones. El porcentaje de limo osciló entre 43,02\% (est.14) y 67,07\% (est.13) con una media de 55,22\% . La arcilla varió entre $32,93 \%$ (est. 13) y $56,98 \%$ (est. 14 ) con un porcentaje promedio de $44,78 \%$. La distribución espacial de las clasificaciones texturales, mostró que las fracciones más finas (arcilla) están asociadas principalmente a las cabeceras protegidas de los canales más directamente influidos por el sistema glacial (cabeceras del estrecho Baker y los senos Eyre, Europa y estero Peel). En cambio, las texturas más gruesas (limo muy fino) se determinaron hacia el occidente, en las bocas expuestas (Baker, canal Messier, paso del Indio, senos Penguin y Europa, y canal Amalia).

Tabla 2. Porcentajes de componentes texturales, materia orgánica total, clasificación textural, y color en los sedimentos.

Table 2. Size fraction percentages, total organic matter, textural classification and color of sediments.

\begin{tabular}{|rrrrll|}
\hline Estación & $\begin{array}{c}\text { Limo } \\
(\boldsymbol{\%})\end{array}$ & $\begin{array}{c}\text { Arcilla } \\
(\boldsymbol{\%})\end{array}$ & $\begin{array}{c}\text { M.O.T } \\
(\boldsymbol{\%})\end{array}$ & Textura & Color \\
\hline 1 & 58,16 & 41,84 & 5,91 & Limo muy fino & Gris oliva (5Y4/1) \\
2 & 57,22 & 42,78 & 4,43 & Limo muy fino & Gris pardo (5YR4/1) \\
3 & 45,91 & 54,09 & 4,21 & Arcilla & Gris oliva (5Y4/1) \\
4 & 57,04 & 42,96 & 7,02 & Limo muy fino & Gris oliva claro (5Y6/1) \\
5 & 64,22 & 35,78 & 4 & Limo muy fino & Gris oliva (5Y4/1) \\
6 & 58,59 & 41,41 & 5,92 & Limo muy fino & Gris oliva (5Y4/1) \\
7 & 56,82 & 43,18 & 9,19 & Limo muy fino & Gris oliva (5Y4/1) \\
8 & 46,12 & 53,88 & 4,77 & Arcilla & Gris verde oscuro (5GY4/1) \\
9 & 44,28 & 55,72 & 2,47 & Arcilla & Gris medio oscuro (N4) \\
10 & 53,59 & 46,41 & 2,9 & Limo muy fino & Gris oliva (5Y4/1) \\
11 & 62,76 & 37,24 & 4,61 & Limo muy fino & Gris oliva (5Y4/1) \\
12 & 63,98 & 36,02 & 5,01 & Limo muy fino & Gris oliva (5Y4/1) \\
13 & 67,07 & 32,93 & 3,3 & Limo muy fino & Gris oliva claro (5Y6/1) \\
14 & 43,02 & 56,98 & 2,5 & Arcilla & Gris oliva (5Y4/1) \\
15 & 43,85 & 56,15 & 3,79 & Arcilla & Gris pardo (5YR4/1) \\
16 & 49,75 & 50,25 & 2,45 & Arcilla & Gris oliva claro (5Y6/1) \\
17 & 66,34 & 33,66 & 2,85 & Limo muy fino & Gris amarillento (5Y8/1) \\
\hline
\end{tabular}


En las Figs. 3 a la 7 y en la Tabla 3, se observan las distribuciones granulométricas y los parámetros de tendencia central y dispersión en las 17 estaciones. El tamaño medio de las partículas osciló entre 8,12 f (est. 14) y 7,67 f (est. 17) con un promedio de 7,89f . Este parámetro se distribuyó espacialmente presentando los valores más altos (diámetro menor), respecto al promedio, en las cabeceras de los canales interiores (cabeceras del Baker, Eyre, Falcon, Europa y Peel). Los diámetros mayores estuvieron asociados a las bocas expuestas del estrecho Baker, canal Messier, seno Penguin, seno Europa y fiordo Amalia).

Respecto al parámetro de selección (Tabla 3), estuvo comprendido de acuerdo a la clasificación
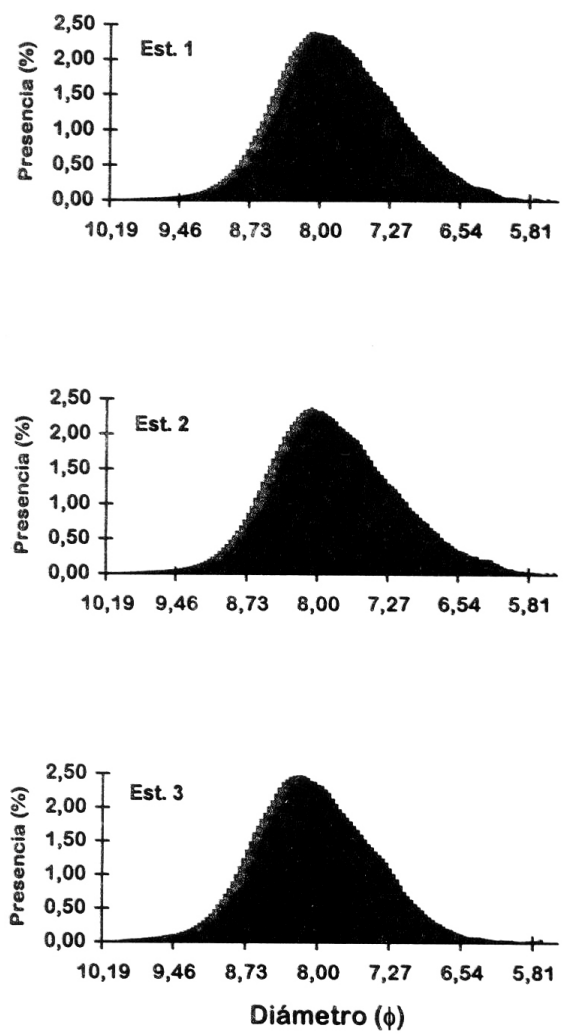

Figura 3. Distribución granulométrica de los sedimentos en el Estrecho Baker.

Figure 3. Grain-size distribution of sediments in the Estrecho Baker.
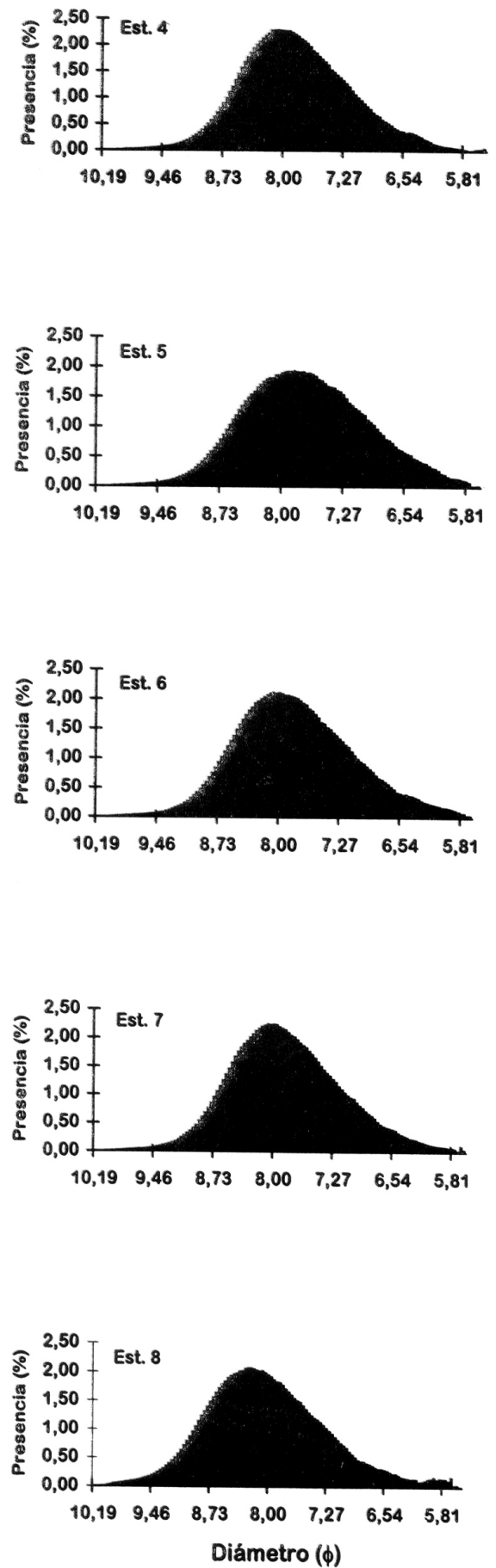

Figura 4. Distribución granulométrica de los sedimentos en el Canal Messier.

Figure 4. Grain-size distribution of sediments in the Canal Messier. 
de Folk (1980), entre sedimentos moderadamente bien seleccionados $(0,62$ f; est. 3 , est. 10$)$ y moderadamente seleccionados $(0,82 \mathrm{f}$; est. 15$)$, con un promedio de $0,69 f$. Observando el comportamiento espacial de selección, es posible reconocer que los valores más bajos (mejor seleccionados) se localizaron preferentemente en el Baker y cabeceras del Eyre, Falcon y Europa. Los sedimentos con peor selección se ubicaron a lo largo del Messier proyectándose, a través del paso del Indio, hasta la boca del seno Europa, más al sur todas las estaciones presentaron esta misma tendencia.

El parámetro de asimetría en los sedimentos, tuvo un predominio de valores negativos, es decir, la
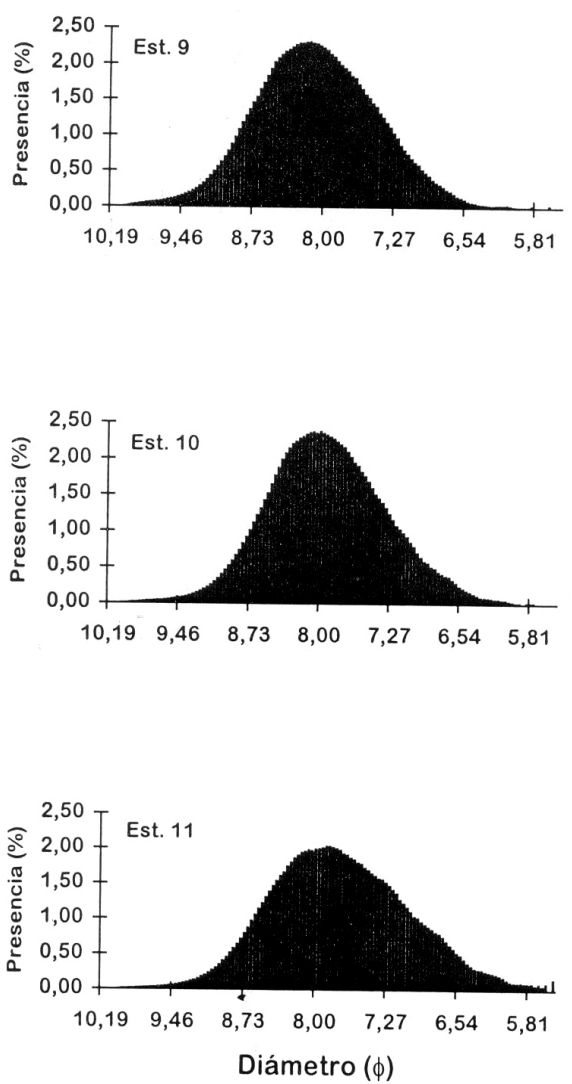

Figura 5. Distribución granulométrica de los sedimentos en los Senos Eyre, Falcon y Penguin.

Figure 5. Grain-size distribution of sediments in the Seno Eyre, Falcon and Penguin. mayoría de las muestras incluyeron un exceso de gruesos, oscilando entre $-0,47$ (est. 8 ) y 0,02 (est. 16), con un promedio de -0,23. El Baker, Messier, paso del Indio y bocas del Penguin y Europa contienen los sedimentos con mayor asimetría negativa, en cambio las cabeceras del Eyre, Falcon, Europa, Peel y Amalia presentaron una tendencia a las asimetrías menos negativas $(>-0,23)$.

La curtosis se presentó con valores extremos de 2,71 (est. 15) y 3,69 (est. 3), con un promedio de 3,05. De acuerdo a la clasificación de Folk (1980) variaron entre sedimentos muy leptocúrticos y extremadamente leptocúrticos, indicando predominio de distribuciones agudas. Respecto al promedio de
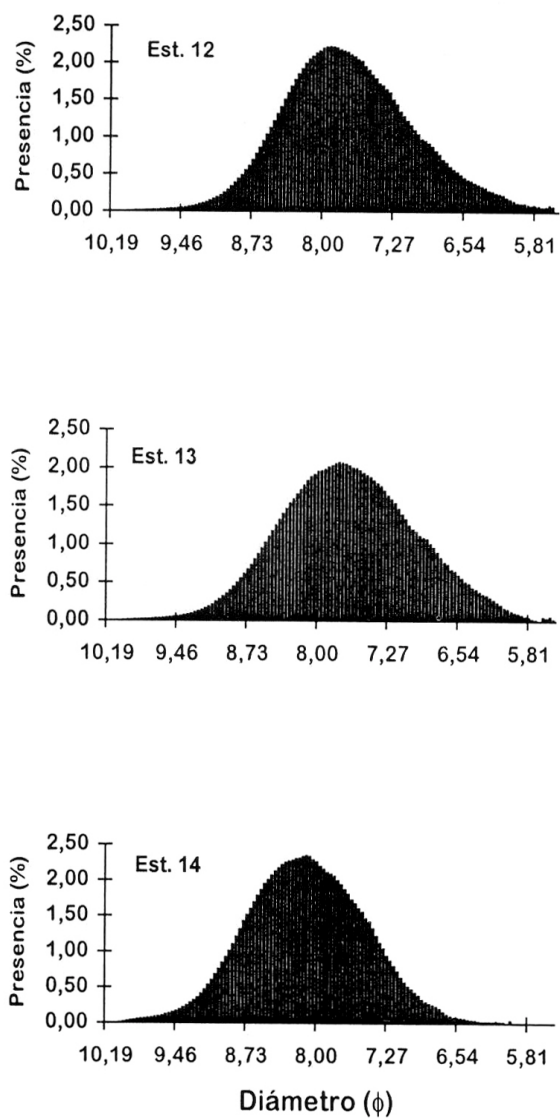

Figura 6. Distribución granulométrica de los sedimentos en el Seno Europa.

Figure 6. Grain-size distribution of sediments in the Seno Europa. 
la curtosis, es posible reconocer dos sectores con similar comportamiento cúrtico. El extremo norte (estrecho Baker y boca del Messier) está controlado por sedimentos con una mayor tendencia al leptocurtismo $(>3,05)$ en cambio el extremo sur (Europa, Peel, Amalia y canal Inocentes) contiene sedimentos con una menor tendencia $(<3,05)$.

La presencia de materia orgánica total (MOT) en los sedimentos de los fiordos y canales (Tabla 2), osciló entre $2,45 \%$ (est. 16) y 9,19\% (est. 7), con un promedio de $4,43 \%$. El comportamiento espacial indicó que los valores más bajos (inferiores a $4,43 \%$ ), están concentrados en los sectores de las cabeceras interiores cercanas al glaciar (cabeceras
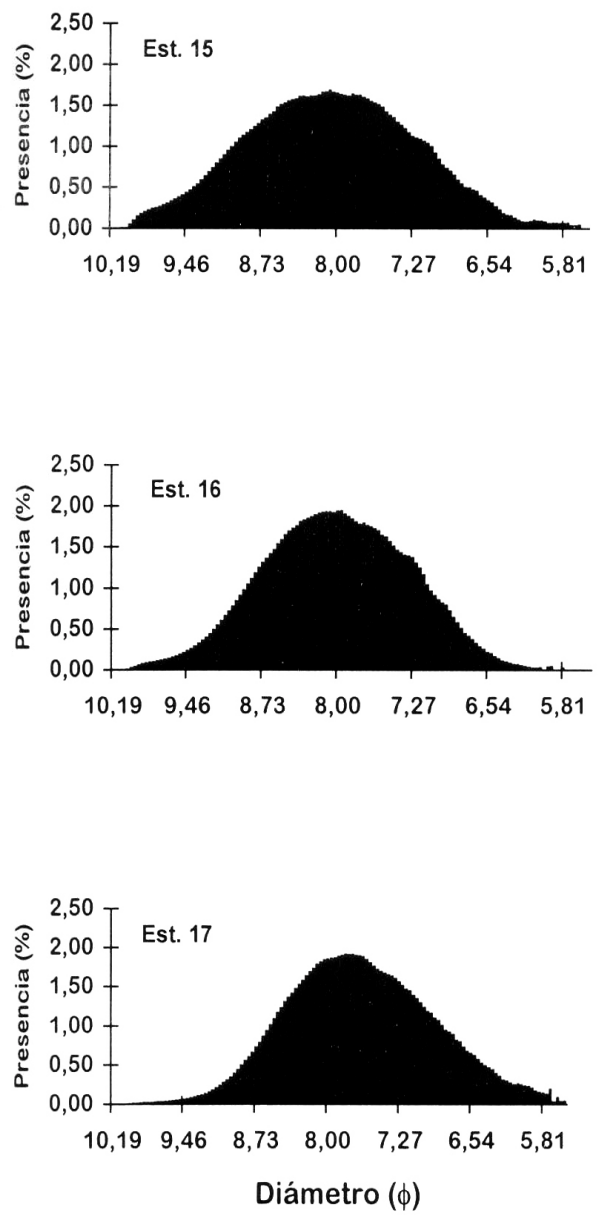

Figura 7. Distribución granulométrica de los sedimentos en el Canal Inocentes, Estero Peel y Fiordo Amalia.

Figure 7. Grain-size distribution of sediments in the Canal Inocentes, Estero Peel and Fiordo Amalia. del Baker, Eyre, Falcon, Europa y Peel). Por otro lado, los mayores porcentajes (superiores a 4,43\%) se distribuyeron principalmente en los canales más expuestos y próximos al mar (bocas del Baker, Messier, Penguin, Europa y paso del Indio).

El color de los sedimentos de los fiordos y canales (Tabla 3), se clasificaron de acuerdo a la Carta de colores (Goddard, 1975), en grises muy claros (N8) con tonalidad gris amarillenta (5Y8/1), grises medianamente claros (N6) con tonalidad gris oliva clara $(5 \mathrm{Y} 6 / 1)$ y grises medianamente oscuros (N4) con tonalidades gris café (5YR4/1), gris oliva (5Y4/ 1) y gris verde oscuro $(5 G Y 4 / 1)$. En cuanto a la distribución espacial de la coloración, se reconoció un predominio de los sedimentos grises medianamente oscuros, especialmente en los canales más expuestos y próximos al mar.

Tabla 3. Parámetros granulométricos de los sedimentos muestreados.

Table 3. Statistical grain-size parameters of sampled sediments.

\begin{tabular}{|ccccl|}
\hline Estación & $\begin{array}{c}\text { Media } \\
\text { (f) }\end{array}$ & $\begin{array}{c}\text { Selección } \\
\text { (f) }\end{array}$ & Asimetría & Curtosis \\
\hline 1 & 7,86 & 0,63 & $-0,23$ & 3,1 \\
2 & 7,86 & 0,65 & $-0,3$ & 3,11 \\
3 & 8,05 & 0,62 & $-0,35$ & 3,69 \\
4 & 7,86 & 0,66 & $-0,3$ & 3,11 \\
5 & 7,71 & 0,74 & $-0,26$ & 2,9 \\
6 & 7,81 & 0,72 & $-0,34$ & 3,06 \\
7 & 7,85 & 0,69 & $-0,36$ & 3,19 \\
8 & 8,03 & 0,76 & $-0,47$ & 3,34 \\
9 & 8,09 & 0,63 & $-0,09$ & 3,04 \\
10 & 7,94 & 0,62 & $-0,17$ & 3,1 \\
11 & 7,75 & 0,72 & $-0,25$ & 2,94 \\
12 & 7,74 & 0,69 & $-0,33$ & 3,17 \\
13 & 7,69 & 0,7 & $-0,11$ & 2,85 \\
14 & 8,12 & 0,61 & $-0,04$ & 3,01 \\
15 & 8,07 & 0,82 & $-0,11$ & 2,71 \\
16 & 8,03 & 0,71 & 0,02 & 2,72 \\
17 & 7,67 & 0,75 & $-0,19$ & 2,81 \\
\hline
\end{tabular}




\section{DISCUSION}

El sistema de fiordos y canales adyacentes a Campos de Hielo Sur, se comporta de acuerdo a la regla de predominancia textural limosa, descrita para este tipo de ambientes en altas latitudes (Pickrill, et al., 1981; Skei y Melsom, 1982; Bogen, 1983). Según Seibold y Berger (1993), la presencia del limo estaría controlada por la meteorización mecánica del relieve continental, a través del ciclo congelamientodescongelamiento. La aparente carencia de fracciones más gruesas (arena y grava), podría ser explicada por el modelo de distribución espacial de las texturas propuesto por Piper et al. (1983), en el cual las fracciones más gruesas están ubicadas en los sectores costeros de los fiordos a profundidades inferiores a $15 \mathrm{~m}$. En cambio, las fracciones finas se localizan en los sectores más profundos. Por lo anterior, de acuerdo a la localización de las estaciones del presente muestreo (centro del canal), parece lógica la obtención sólo de sedimentos finos.

De acuerdo a la distribución espacial de las texturas, es posible reconocer el predominio de arcillas en las cabeceras de los fiordos y de limos en las bocas de los canales. Este comportamiento respondería, por una parte, a las características morfológicas y de localización de las cabeceras, que las convierten en ambientes protegidos con baja energía cinética (menor influencia de las olas y corrientes de marea), permitiéndose la decantación de las fracciones más finas. Además, la acción de las mareas genera, en este tipo de ambientes, un transporte selectivo de arcillas en dirección a las cabeceras (Clifton, 1982). Por estas mismas razones, las fracciones relativamente más gruesas (limos) se encuentran en los sectores más expuestos al accionar marino, donde el proceso de remoción del particulado fino por el «retrabajo» de olas y corrientes de marea es mayor (Reineck y Singh, 1980).

El tamaño medio de las partículas del sedimento refleja las condiciones hidrodinámicas (energéticas) que actuaron en su transporte y depositación (Folk, 1980). Generalmente, las partículas más gruesas están asociadas a un mayor nivel de energía, en cambio, las finas se encuentran en sectores con menor hidrodinamismo. De este modo, el reconocimiento de partículas más finas en las cabeceras indica que se trata de ambientes de energía relativamente baja por el contrario, las bocas, con tamaños medios mayores, tendrían una alta energía asociada.
En términos generales, la selección de los sedimentos evidencia la intensidad de las corrientes que operaron sobre el depósito, es decir, una buena selección se logra cuando actúa una corriente (con alta o baja energía), de intensidad relativamente constante. Se observan malas selecciones cuando actúan procesos dinámicos con amplia fluctuación (Folk, 1980). De este modo, la distribución del parámetro de selección en el sistema de fiordos y canales, indica que el Baker y cabeceras del Eyre, Falcon y Europa están sometidos a corrientes con intensidad intermedia o de menor fluctuación energética. Asimismo, es posible reconocer que el canal Messier, Paso del Indio, bocas del Penguin y Europa, tienden a presentarse como una unidad sometida a una mayor variabilidad de sus procesos dinámicos. Esta variabilidad podría estar relacionada con la fluctuación de las corrientes mareales, ya que los mayores rangos de mareas de sicigia $(1,74-2,40 \mathrm{~m})$, se encuentran en el canal Messier y paso del Indio (SHOA, 1996).

El parámetro de asimetría indica la importancia de las «colas» en la distribución granulométrica, mostrando los excesos de partículas en los extremos de la curva. El significado dinámico para el área de estudio, donde predominan valores negativos, se podría atribuir a la existencia de corrientes que han operado durante un período con una mayor energía a la normal, provocando el aporte de partículas más gruesas (Sahu, 1964). Del mismo modo, Duane (1964) relaciona las tendencias negativas con un alto nivel energético produciendo la erosión de los finos, en cambio, los índices positivos indicarían bajos niveles de hidrodinamismo.

Sagga (1992), asocia las fluctuaciones de la asimetría con las variaciones de la energía física de los diferentes agentes dinámicos. Así es posible sugerir, de acuerdo a la distribución espacial del parámetro, que las corrientes mareales que actúan a lo largo del canal Messier, paso del Indio, y hacia el sur, expliquen el predominio negativo de la asimetría, mediante la erosión del particulado más fino. Nuevamente, se reconoce al canal localizado entre las estaciones 4 a la 12 (canal Messier, paso del Indio, bocas de los senos Penguin y Europa) como una unidad sedimentológica controlada por procesos dinámicos similares. En cambio, las cabeceras parecen estar dominadas por procesos depositacionales del material más fino debido a su relativamente bajo nivel energético.

Respecto al parámetro de curtosis no es posible 
reconocer tendencias e interpretaciones espaciales en el sistema de fiordos y canales adyacentes a Campos de Hielo Sur. Sin embargo, es factible proponer que las tendencias leptocúrticas, es decir, curvas unimodales, podrían explicarse por el predominio de una fuente sedimentaria, probablemente limos generados mediante la acción glacial.

De acuerdo a Friedman y Sanders (1978), los sedimentos de los fiordos con aguas estancadas, presentan altos contenidos de MOT; en cambio en los sistemas ventilados, los porcentajes son menores. La ventilación, que depende de la profundidad del umbral, controla la presencia de MOT en los sedimentos, debido a que las aguas ventiladas contienen una mayor proporción de oxígeno capaz de oxidar los elementos orgánicos. En cambio, con deficiente ventilación, la concentración de oxígeno disminuye, reduciéndose la tasa de oxidación de la MOT, favoreciendo su acumulación.

Considerando que los sedimentos con mayor presencia de MOT y más oscuros del sistema (grises medianamente oscuros; N4), se localizan en los canales expuestos y próximos al mar, se reconoce que el área de estudio se ajusta a la regla que asocia la MOT a la coloración de los sedimentos (Reineck y Singh, 1980). De acuerdo a lo anterior, y sobre la base de los resultados obtenidos de MOT y coloración de los fiordos y canales, se podría sugerir que el sistema, en general, está sujeto a una renovación relativamente periódica de sus aguas produciendo condiciones subóxicas. Este resultado coincide con la observación hecha por Pickard (1971), respecto a la baja presencia de cuencas estancadas, debido principalmente a la ausencia de umbrales someros.

Se sugiere, además, que los aportes de MOT no provienen desde el continente, puesto que las fracciones finas (arcillas), ubicadas en las cabeceras, contienen bajos porcentajes de MOT y coloración más clara. Si el continente aportara la MOT, los elementos orgánicos deberían sedimentar en conjunto con las partículas finas inorgánicas en la cabecera (Seibold y Berger, 1993). De este modo, se sugiere que la fuente de MOT, para el área de estudio, proviene principalmente de la productividad marina, puesto que el sector marino presenta la coloración más oscura y la mayor presencia de MOT. Así, el continente aportaría, principalmente, materiales minerales, producidos por la acción glaciar, que disminuyen la abundancia relativa de la MOT en los sedimentos de las cabeceras.

\section{CONCLUSIONES}

1. En el sistema de fiordos y canales adyacentes a Campos de Hielo Sur predominan las texturas limosas en sus fondos blandos. Estos limos probablemente son el resultado de la acción glacial sobre el continente.

2. De acuerdo a la clasificación textural, tamaño medio, selección y asimetría de los sedimentos, se reconoce la existencia de dos grandes zonas de energía cinética. En el sector este, las cabeceras protegidas directamente influenciadas por el glaciar, presentan sedimentos con características arcillosas, menor tamaño medio, mejor selección y asimetrías menos negativas, indicando la menor energía del proceso sedimentario. En el sector oeste, los canales más expuestos a la acción marina contienen sedimentos limosos, con un mayor tamaño medio, peor selección y asimetrías más negativas, deduciéndose la participación de una mayor energía cinética.

3. El canal Messier, paso del Indio, bocas de los senos Penguin y Europa se comportan, de acuerdo a los parámetros granulométricos (tamaño medio, selección y asimetría) y texturales, como una sola unidad sedimentaria probablemente controlada por las corrientes de mareas.

4. De acuerdo a los contenidos de MOT y coloración de los sedimentos, se infiere que el sistema de fiordos y canales está sujeto a una renovación periódica de sus aguas, generando un ambiente subóxico.

5. De acuerdo a los resultados sedimentológicos y a la distribución de MOT se concluye que los aportes de MOT provienen principalmente del sector marino. En cambio, el sector continental aportaría sólo las fracciones minerales al sistema, disminuyendo el contenido orgánico relativo en las cabeceras protegidas.

\section{AGRADECIMIENTOS}

Este estudio fue financiado parcialmente por el Servicio Alemán de Intercambio Académico (DAAD) y proyecto DIUC 96.310.021-1. Los autores agradecen muy especialmente, en orden alfabético, la colaboración de Alberto Araneda, Oscar Retamal, Dr. José Stuardo, Jorge Valdés y Patricia Virano. 


\section{REFERENCIAS}

Bogen, J. 1983. Morphology and sedimentology of deltas in fjord and fjord valley lakes. Sediment. Geol., 36: 245-267.

Börgel, R. 1983. Geografía de Chile. Geomorfología. Instituto Geográfico Militar, Santiago, 182 pp.

Clifton H.E. 1982. Estuarine deposits. In: P.A. Scholle y D.R. Spearing (eds.), Sandstone depositional environments. The American Association of Petroleum Geologists, Tulsa, Oklahoma, pp. 179190.

Comité Oceanográfico Nacional (CONA). 1996. Informe de Crucero. Crucero de investigación científica marina a los fiordos y canales adyacentes a Campos de Hielo Sur. Comité Oceanográfico Nacional, Valparaíso, $122 \mathrm{pp}$.

Duane, D.B. 1964. Significance of skewness in recent sediments, western Pamlico Sound, North Carolina. J. Sed. Petrol., 34(4): 864-874.

Folk, R.L. 1980. Petrology of sedimentary rocks. Hemphill, Austin,Texas, 182 pp.

Friedman, G.M. y J.E. Sanders. 1978. Principles of sedimentology. John Wiley \& Sons, New York, 792 pp.

Goddard, E.N. 1975. Rock-color chart. Geological Society of America, Boulder, Colorado.

González, G., J. Muñoz y J. Barboza. 1987. Geografía de Chile. Geografía XII Región de Magallanes y de la Antártica Chilena. Instituto Geográfico Militar, Santiago, 368 pp.

Mills, A. 1978. A comparison of methods of determining organic carbon in marine sediments with suggestions for a standard method. Hydrobiologia, 57(1): 45-52.

Pickard, G.L. 1971. Some physical oceanographic features of inlets of Chile. J. Fish. Res. Bd. Canada, 28(8): 1077-1106

Recibido el 26 de mayo de 1997.

Aceptado el 24 de abril de 1998.
Pickrill, R.A., J. Irwin y B.S. Shakespeare. 1981. Circulation and sedimentation in a tidal-influenced fjord lake: Lake McKerrow, New Zealand. Estuar. Coast. Shelf Sci., 12 (1): 23-37.

Piper, D.J.W., J.R. Letson, A.M. de Iure y C.Q. Barrie. 1983. Sediment accumulation in lowsedimentation, wave dominated, glaciated inlets. Sediment. Geol., 36: 195-215.

Pritchard D.W. 1976. La circulación y la mezcla en las regiones costeras y los estuarios. In: R.C. Vetter (ed.). Oceanografía, la última frontera. Editorial El Ateneo, Buenos Aires, pp. 273-282.

Reineck, H. E. y I.B. Singh. 1980. Depositional sedimentary environments. Springer-Verlag, Berlin, 549 pp.

Sagga, A.M.S. 1992. The use of textural parameters of sand in studying the characteristics and depositional processes of coastal sediments on Saudi Arabian beaches. Mar. Geol., 104: 179-186.

Sahu, B. 1964. Depositional mechanism from the size analysis of clastic sediments. J. Sed. Petrol., 34: 73-83.

Seibold, E. y W.H. Berger. 1993. The sea floor. An introduction to marine geology. Springer-Verlag, Berlin, 355 pp.

Servicio Hidrográfico y Oceanográfico de la Armada de Chile (SHOA). 1996. Tablas de marea de la costa de Chile y puntos de la costa sudamericana. SHOA, Valparaíso, 337 pp.

Seward-Thompson, B.L. y J.R. Hails. 1973. An appraisal of the computation of statistical parameters in grain size analysis. Sedimentology, 20: 161-169.

Skei, J.M. y S. Melsom. 1982. Seasonal and vertical variations in the chemical composition of suspended particulate matter in an oxygen-deficient fjord. Estuar. Coast. Shelf Sci., 14(1): 61-78.

Wentworth, C.K. 1922. A scale of grade and class terms for clastic sediments. J. Geol., 30: 377-392. 